\title{
Professional quality of life amongst nurses in psychiatric observation units
}

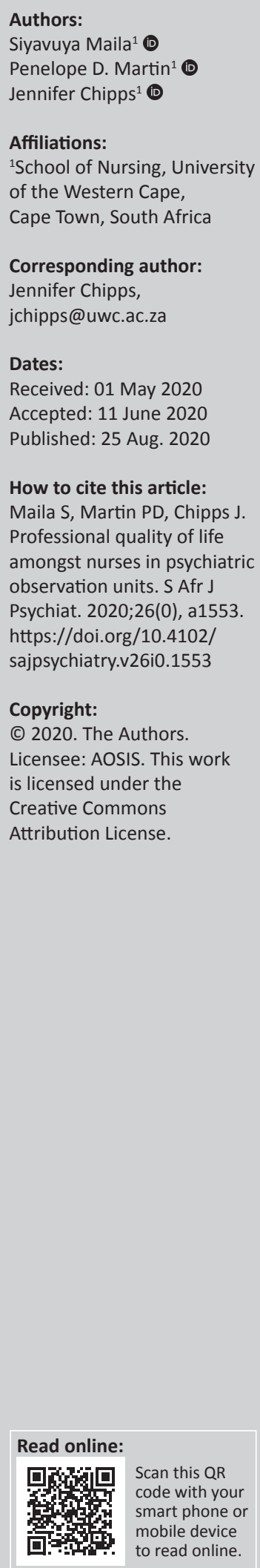

Background: Professional quality of life amongst nurses in psychiatric observations units may be affected by working conditions such as an overflow of mental health care users (MHCUs), a shortage of nurses, lack of specialised staff and inadequate infrastructure to accommodate MHCUs amongst others.

Aim: The aim of the study was to investigate the professional quality of life amongst nurses in psychiatric observation units.

Setting: The study was conducted in psychiatric observation units in eight hospitals in the Metropole District Health Services in the Western Cape.

Method: A quantitative descriptive survey design using the Professional Quality of Life (ProQoL version 5) questionnaire was conducted with an all-inclusive sample of 175 nurses. The ProQoL has two scales, namely, the compassion satisfaction and the compassion fatigue. Compassion fatigue includes two subscales, burnout and secondary traumatic stress. Ethics to conduct the study was obtained from the Research Ethics Committee at the university and the Department of Health in the Western Cape.

Results: A response rate of $93 \%(n=163)$ was obtained. Respondents reported moderate compassion satisfaction. Psychiatric nurse specialists and registered nurses reported lower compassion satisfaction than enrolled nurses and nursing assistants. This came with moderate levels of burnout and high levels of secondary traumatic stress, with enrolled nurses and enrolled nursing assistants reporting lower levels than the other professional groups.

Conclusion: Psychiatric nurse specialists and registered nurses experienced higher burnout and secondary traumatic stress and lower compassion satisfaction than the lower categories of nurses.

Keywords: Burnout; Compassion Fatigue; Compassion Satisfaction; Professional Quality of Life; Secondary Traumatic Stress.

\section{Introduction}

South Africa has a high prevalence of mental health problems with $30.3 \%$ reported common mental health problems and a $47.5 \%$ projected risk of mental disorders during a lifetime. ${ }^{1}$ This is concomitant with a large mental health treatment gap of $92 \% .^{2}$ The Mental Health Care Act No. 17 of 2002 aims to bridge this gap by integrating mental healthcare services into primary healthcare, specifically with the creation of 72-h observation units (psychiatric observation units) in selected general hospitals for Mental Health Care Users (MHCUs) requiring involuntary admission. The creation of these 72 -h observation units have contributed to high patient turnover and overflow, longer length of stays and inadequate or limited financial, infrastructure and human resources. ${ }^{3}$ These observation units are often short staffed and manned by non-specialist psychiatric nurses. ${ }^{4}$ In KwaZulu-Natal, almost $70 \%$ of hospitals did not have enough-skilled nursing and medical staff to render required mental health services. ${ }^{3}$

Compassion fatigue impacts on compassion satisfaction (the fulfilment attained from helping and caring for others). ${ }^{5,6}$ Compassion fatigue includes experiences of burnout (feeling drained and emotionally worn-out) ${ }^{7,8}$ and secondary traumatic stress (emotional strain associated with the exposure to stressful, traumatic events and danger at work). ${ }^{5,6}$

Professional quality of life in nurses has been investigated in general and mental health establishments globally, ${ }^{9,10,11,12,13,14}$ and in general health establishments in South Africa. ${ }^{15,16,17,18}$ These studies have found that nurses are predisposed to burnout and secondary traumatic 
stress more than any other healthcare providers because of long hours in health facilities, administrative workload, supervision and staffing challenges. ${ }^{7,19}$ In mental healthcare establishments as in general health, professional quality of life for nurses is an important aspect of career satisfaction and retention. ${ }^{14,20,21}$

Burnout amongst nurses working in psychiatric hospitals are common ${ }^{22}$ and has been attributed to the scarcity of essential requirements in hospitals such as skilled staff, equipment, budgets, ${ }^{23,24}$ high clinical and administrative workloads of nurses, ${ }^{3,7,25}$ exposure to chronic MHCUs ${ }^{26}$ and the prolonged stay of $\mathrm{MHCUs}^{3,25}$ and the prolonged exposure to direct and indirect trauma in the units. ${ }^{27}$ In addition, the nature of acute admissions to 72-h observation units may expose nurses to aggression related to acute illness, ${ }^{13}$ especially during involuntary admissions. ${ }^{20}$

In South Africa, there are four nursing categories ${ }^{28}$ : working in psychiatric observation units; enrolled nursing assistants and enrolled nurses who have no formal education or training in psychiatric nursing apart from in-service training; registered nurses who have completed a 4-year diploma or degree in nursing (general, community and psychiatry) and midwifery; and psychiatric nurse specialists who have an advanced qualification in psychiatric nursing science. ${ }^{28}$ Internationally, registered nurses were reported to have lower secondary traumatic stress than nursing assistants, ${ }^{21}$ although registered nurses working in child and adolescent psychiatry were reported to have higher burnout than nursing assistants. ${ }^{14}$ Patient's needs, type of illnesses and symptom and the administrative roles in the unit and staff shortages were all reported as stressors related to burnout by registered nurses, whilst enrolled nurses and enrolled nursing assistants reported shortage of staff, challenges involved with patient care and lack of acknowledgement for their hard work. ${ }^{29}$

To date, no study has been carried out in South Africa to assess the professional quality of life of these nurse categories, and no study has specifically assessed the specific impact of psychiatric observation units on these nurse categories. This study aimed to investigate the professional quality of life amongst nurses in psychiatric observations units in the Metropole District Health Services in the Western Cape, South Africa.

\section{Research methods and design Study design}

A survey was conducted with all nurse categories.

\section{Setting}

The setting of the study was 16 psychiatric observation units in eight hospitals in the Metropole District Health Services in the Western Cape, South Africa.

\section{Study population and sampling strategy}

The population for this study was 175 nurses (14 psychiatric nurse specialists, 59 registered nurses, 24 enrolled nurses and 78 enrolled nursing assistants), all providing direct nursing care to MHCUs. An all-inclusive sampling strategy was used.

\section{Instrument}

The 30-itemed, 5-point Likert scale, Professional Quality of Life 5 instrument (ProQoL 5) was used (with permission). ${ }^{6}$ The ProQoL 5 is a validated instrument which aims to the assess respondents' feelings or experiences of compassion satisfaction and compassion fatigue in the last 30 days. The ProQoL has two scales, compassion satisfaction and compassion fatigue. Compassion fatigue has two subscales, namely burnout and secondary traumatic stress. ${ }^{6}$ The ProQoL 5 has acceptable reliability and validity with Cronbach's alpha scale reliability for the subscales (for compassion satisfaction, $\alpha=0.88$; for burnout, $\alpha=0.75$, and for secondary traumatic stress, $\alpha=0.81$ ) and has good construct validity in more than 200 articles which have been published. ${ }^{6}$

\section{Data collection}

The researcher visited the eight hospitals to obtain permission to gain access for data collection. Information sheets were handed to all potential respondents to explain the study and to obtain their consent. The data were collected from August 2018 to October 2018.

\section{Data analysis}

Data were analysed using SPSS version 25 (SPSS Inc., Chicago, IL, USA). Demographic characteristics and the ProQoL individual items were analysed using descriptive statistics. The scores for the sub-scales were calculated using the raw scores and using the ProQoL manual's cut points, categories for the low, middle and high scores for compassion satisfaction (score $>42=$ high, between 32 and $42=$ moderate and $<32=$ low $)^{5,6}$; burnout (score $>27=$ high, between 18 and $27=$ moderate, and $<18=10 \mathrm{w})^{5,6}$; and secondary traumatic stress (score $>17=$ high, between 8 and $17=$ moderate, and $<8=$ low) were coded. ${ }^{5,6}$ Differences between the categories of nurses were measured using 95\% confidence interval $(\mathrm{CI})$, chi-square tests $\left(X^{2}\right)$ and independent samples Kruskal-Wallis tests $(K)$. Significance was set at $p<0.05$.

\section{Ethical consideration}

Ethical clearance was obtained from the Biomedical Research Council in the University of the Western Cape (ethics reference number: BM18/5/21) and Western Cape Government Health, reference number: WC-201807_024.

\section{Results}

Of the 175 nurses who participated in the study, 163 submitted completed the questionnaires (93\% response 
rate). Two scales showed adequate internal consistency, with Cronbach's $\alpha$ for compassion satisfaction $\alpha=0.763$ and secondary traumatic stress $\alpha=0.741$. The internal consistency for the burnout scale was lower at $\alpha=0.590$.

\section{Demographics}

Of the 163 respondents, $69.3 \%$ (113) were female respondents and $63.2 \%$ (103) of the respondents reported being single. The average age of the respondents was $37.5( \pm 9.4)$ years. Nearly half of the respondents were enrolled nursing assistants $(68,41.9 \%)$, followed by registered nurses $(60,36.8 \%)$, enrolled nurses $(21,12.9 \%)$ and psychiatric specialist nurses $(14,8.6 \%)$ (Table 1). The average years of experience working as nurses were $7.5( \pm 8.4)$ (median 4 years, ranging from 1 to 35 years). There were significant differences amongst the respondents in the nurse categories in terms of gender, with higher proportions of male psychiatric nurse specialist respondents compared with registered nurses, enrolled nurses and enrolled nursing assistant respondents $10(71.4 \%)$ versus $18(30 \%)$ versus $9(42.9 \%)$ versus $13(19.1 \%)$, respectively $\left(\chi^{2}=16.6, p=0.001\right)$.

Enrolled nurse respondents were significantly younger than psychiatric nurse specialist, registered nurses and enrolled nursing assistant responders (34.6 vs. 41.8 vs. 36.1 vs. 38.6 years, respectively $(K=9.1, p=0.020)$. Enrolled nurse respondents also reported significantly less years of experience than psychiatric nurse specialist, registered nurse and enrolled nursing assistant respondents' (4.0 vs. 9.5 vs. 5.0 vs. 4.0 median) years of experience, respectively $K^{2}=14.2, p=0.027$ ) (Table 1).

\section{Professional quality of life amongst nurses in psychiatric observations units}

Overall, the respondents reported moderate compassion satisfaction (41.6 [CI 95\% 40.7-42.5]), moderate burnout (24.6 [CI 95\% 23.7-25.5]) and high secondary traumatic stress (27.36 [CI 95\% 26.2-28.4]) (Table 2).

\section{Compassion satisfaction}

The respondents reported moderate compassion satisfaction (41.6 [CI 95\% 40.7-42.5]), with the highest rating for I am proud of what I can do to help (4.4 [CI 95\% 4.2-4.5]) and the lowest for I feel invigorated after working with those I nurse (3.3 [CI 95\% 3.1-3.3]) (Table 3). These differences were because of enrolled nursing assistants and enrolled nurse respondents rating the following statements significantly higher: I am happy I chose to do this work (4.7 vs. $4.5, K=25.1, p<0.001)$; and I am pleased with how I am able to keep up with nursing techniques and protocols (4.3 vs. $4.2, K=8.6, p=0.034$ ) (Table 3 ).

\section{Burnout}

Overall, the respondents reported moderate burnout scores (24.6 [CI 95\% 23.7-25.5]), with the highest rating for I feel

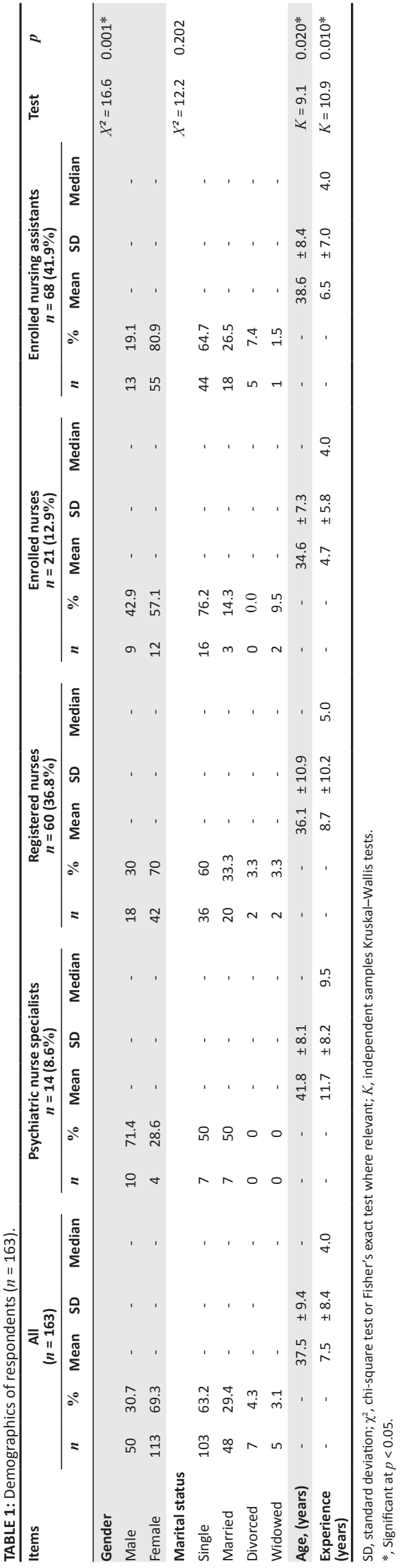



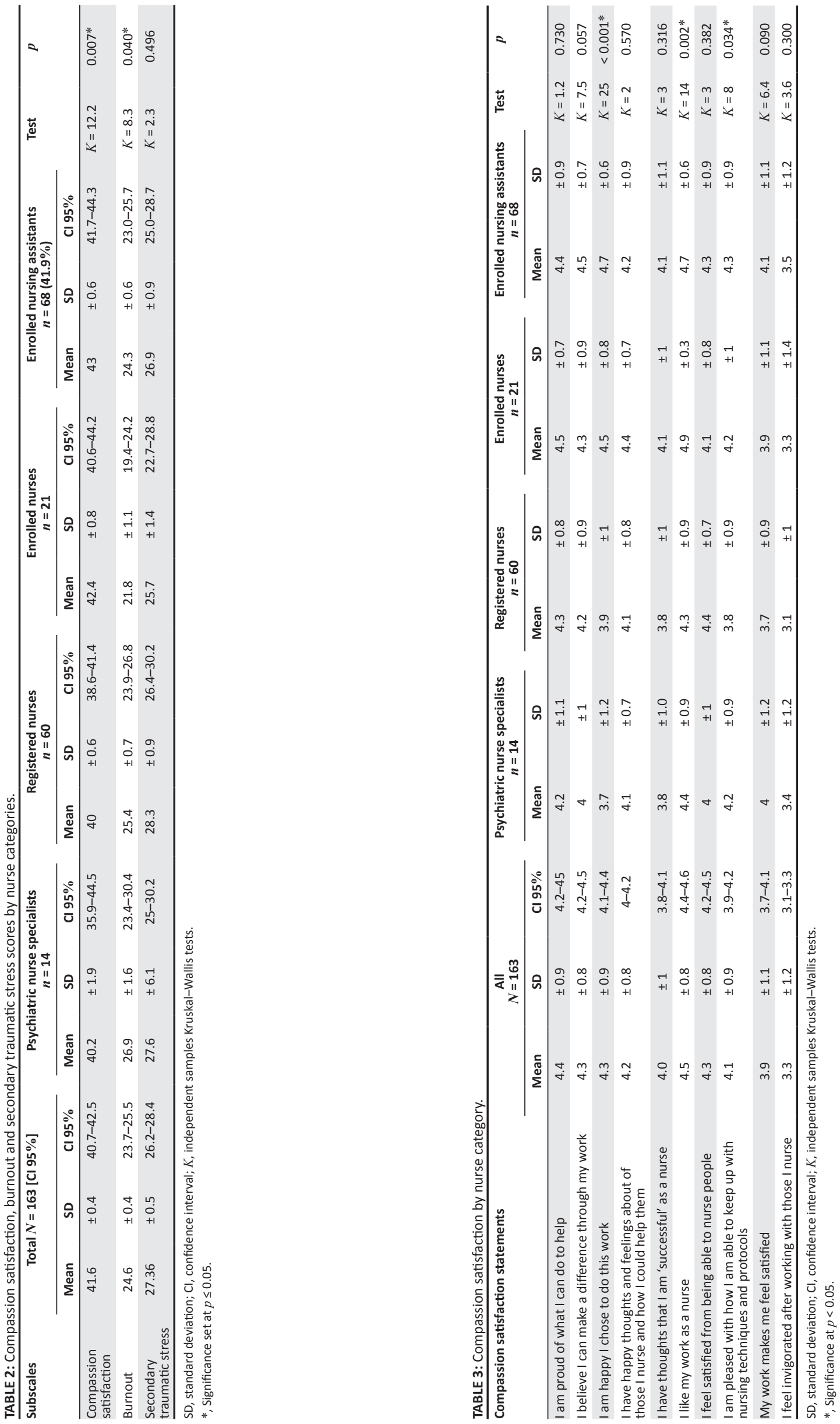
overwhelmed because my case workload seems endless (3.2 [CI 95\% 3-3.4]) and the lowest rating for I am a very caring person (1.6 [CI 95\% 1.5-1.8]) (Table 4). I feel 'bogged' down by the system (3.7 vs. 3.3 vs. 2.6 and $3, \chi^{2}=8.3 p=0.039 *$ ), I feel trapped in my job as a nurse ( 3.0 vs. 2.3 vs. 1.7 vs. $1.9, \chi^{2}=10.6 p=0.014^{*}$ ) and I feel overwhelmed because in my case work load seems endless ( 4 vs. 3.3 vs. 2.6 vs. $3.2, K=9, p=0.028^{*}$ ) were rated significantly higher by the psychiatric nurse specialist than the registered nurse, enrolled nurse and enrolled nursing assistant respondents (Table 4).

\section{Secondary traumatic stress}

The respondents reported high secondary traumatic stress scores (27.36 [CI 95\% 26.2-28.4]), with the highest rating for I am preoccupied with more than one person I nurse (3.5 [CI 95\% 3.3-3.7]) and the lowest for I feel depressed because of traumatic experiences of the people I nursed (2.4 [CI 95\% 2.2-2.5]) (Table 5). There were no overall significant differences between respondents nurse categories (Table 2). I think that I might have been affected by the traumatic stress of those I nurse was rated higher by psychiatric nurse specialist respondents compared with registered nurses and enrolled nursing assistant respondents ( 2.7 vs. 2.4 vs. 1.7 vs. 2.2 vs. $K=6.8 p=0.077)$, although not significant (Table 5).

\section{Discussion}

Overall, all the respondents reported moderate compassion satisfaction, moderate burnout but high secondary traumatic stress. Enrolled nursing assistant respondents had significantly higher compassion satisfaction scores than the other nursing categories, and these respondents reported that they were happy working with MHCUs and felt that they were able to keep abreast of nursing techniques in rendering care for MHCUs. The higher compassion satisfaction in enrolled nursing assistants may be because of the significantly shorter years of experience (median 4.0 years) compared with the psychiatric nursing specialists (median 9.5 years). Although no studies have been carried out on professional quality of life in these different categories of nurses working in mental health establishments, studies in South Africa in a maternity setting in contrast found that registered nurses reported higher compassion satisfaction than enrolled nurses. ${ }^{15}$

In this study, all respondents reported high secondary traumatic stress. The high levels of secondary stress and burnout may be because of the nature of mental healthcare which includes being exposed to the trauma associated with mental illness, ${ }^{4}$ frequent involuntarily admissions of MHCUs $^{4}$ and exposure to aggressive behaviour from MHCUs. ${ }^{30}$ Other studies have also reported the high secondary traumatic stress related to being assaulted by MHCUs. ${ }^{13,31,32}$

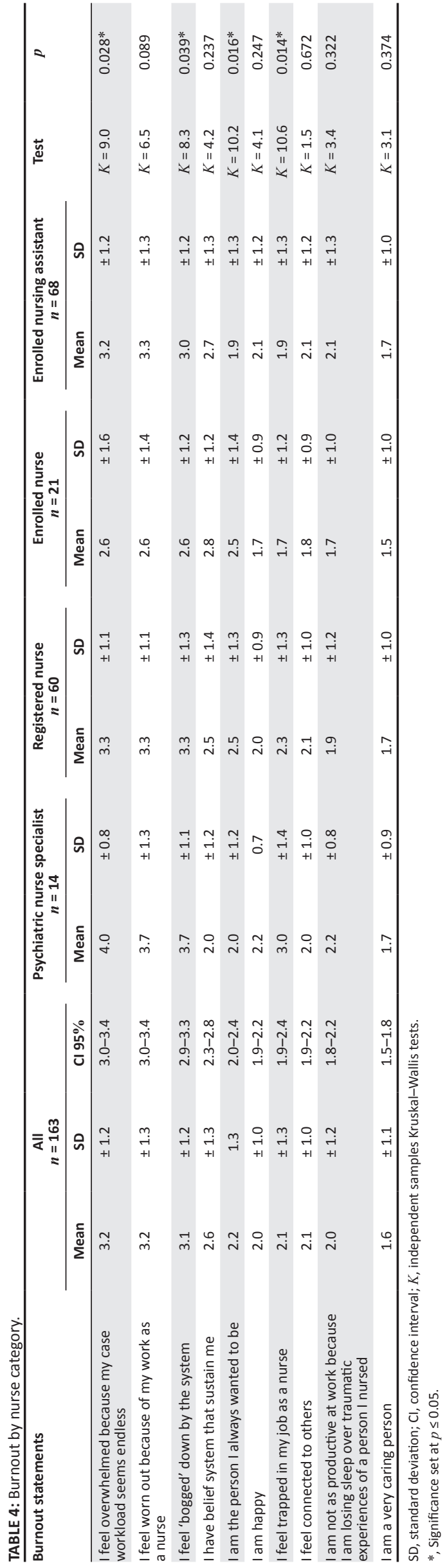




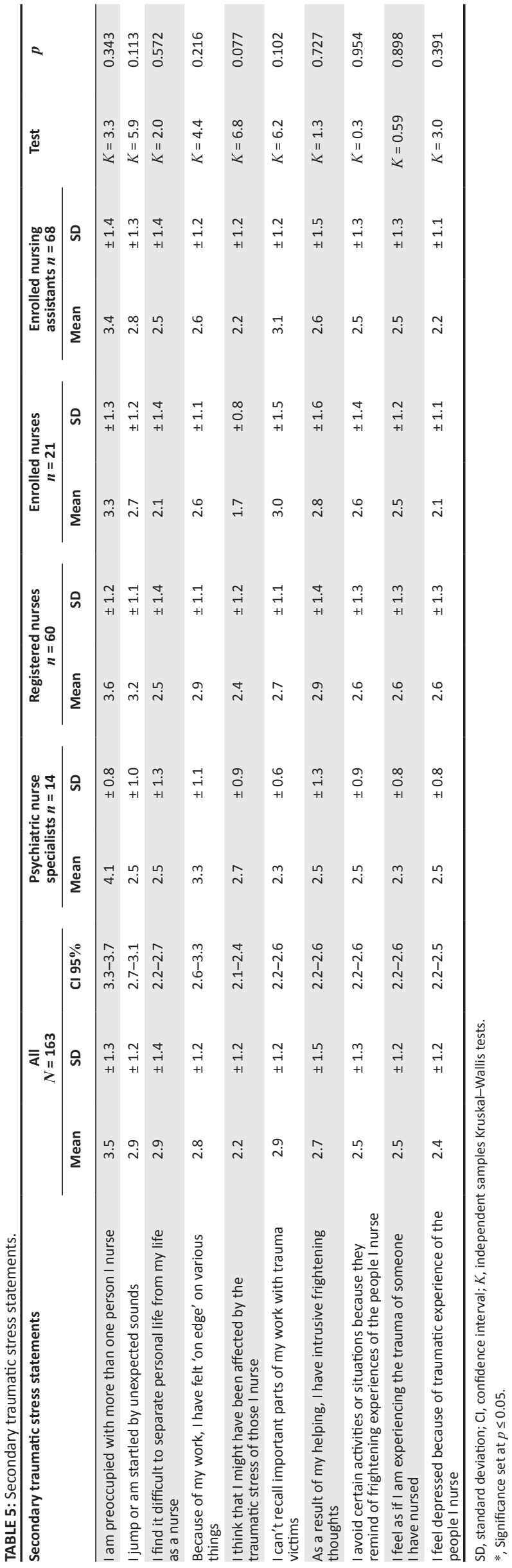

Psychiatric nurse specialist respondents reported higher burnout than the other nursing categories and reported feelings of being bogged down, trapped, overwhelmed and preoccupied with the MHCUs. The higher ratings of burnout by specialist psychiatric nurse respondents specifically may be related to the expectations placed on specialist psychiatric nurses who are expected to render specialist mental healthcare, treatment and rehabilitation commensurate with their education and training. ${ }^{3,4}$ One of the roles of psychiatric nurse specialists is to render psychotherapeutic interventions to MHCUs and the therapeutic process of listening to traumatic experiences and employing empathy may predispose them to secondary trauma. ${ }^{33,34,35}$ Similar findings were reported in Northern England where higher secondary traumatic stress and burnout were found in registered mental health nurses compared with healthcare assistants (similar to enrolled nurse assistants). ${ }^{14}$ These reports in mental health are similar to trends in other health settings such as maternity where registered nurses had higher ratings of compassion fatigue than enrolled and nursing assistants. ${ }^{15}$

A second factor which may contribute to differences in burnout in the nurse categories may be because of differences in professional educational preparation in these categories. A lack of formal psychiatric education and training has been identified as a predisposition to emotional and physical exhaustion, ${ }^{36}$ which can lead to secondary traumatic stress and/or burnout. In contrast, a study in Greece reported higher secondary traumatic stress amongst nurse assistants than registered nurses which they attributed to nurse assistants having more direct contact with MHCUs and less psychiatric training. ${ }^{21}$

Lastly, working conditions such as poor infrastructure and high workload experienced in psychiatric observation units are also thought to contribute to secondary traumatic stress. The psychiatric nurse specialists and registered nurses in these units are expected to lead and be accountable for the management of acutely disturbed MHCUs, and the support from a multi-disciplinary team may be limited, ${ }^{4}$ as specialised staff such as psychiatrists, psychologists, social workers and occupational therapists are scarce. ${ }^{3,4}$

\section{Conclusion}

Psychiatric nurse specialists and registered nurses experienced higher burnout and secondary traumatic stress and lower compassion satisfaction than the other categories of nurses. Professional quality of life for nurses is an important aspect of career satisfaction and retention, ${ }^{37}$ and the on-going investigation of professional quality of life of all nurse categories working in mental health settings is essential..$^{38}$ This is especially important for specialist psychiatric nurses to ensure job satisfaction and to retain an experienced workforce for mental healthcare. 


\section{Acknowledgements Competing interests}

The authors have declared that no competing interests exist.

\section{Authors' contributions}

S.M. contributed to the data collection; S.M. and J.-A.C. performed the data analysis; S.M., P.M. and J.-A.C. contributed to the manuscript preparation.

\section{Funding information}

S.M. was awarded Ada Bertie Levenstein bursary by University of the Western Cape for the Master of Nursing Education Degree (amount paid R 50000.00).

\section{Data availability statement}

Data will be available from the corresponding author upon reasonable request.

\section{Disclaimer}

The views and opinions expressed in this article are those of the authors and do not necessarily reflect the official policy or position of any affiliated agency of the authors.

\section{References}

1. Herman AA, Stein DJ, Seedat S, Heeringa SG, Moomal H, Williams DR. The South African Stress and Health (SASH) study: 12-month and lifetime prevalence of common mental disorders. S Afr Med J. 2009;99(5):339-344. https://doi.org/ 10.7196/SAMJ.3374

2. Docrat S, Besada D, Cleary S, Daviaud E, Lund C. Mental health system costs, resources and constraints in South Africa: A national survey. Health Policy Plan. 2019;34(9):706-719. https://doi.org/10.1093/heapol/czz085

3. Ramlall S, Chipps J, Mars M. Impact of the South African Mental Health Care Ac No. 17 of 2002 on regional and district hospitals designated for mental health care in KwaZulu-Natal. 2010;100(17):2007-2010.

4. Thomas E, Cloete KJ, Kidd M, Lategan H. A decentralised model of psychiatric care Profile, length of stay and outcome of mental healthcare users admitted to a district-level public hospital in the Western Cape. S Afr J Psychiatry. 2015;21(1):8-12. https://doi.org/10.7196/SAJP.538

5. Stamm BH. The Pro-QOL manual: The professional quality of life: Compassion satisfaction and Fatigue Scale Version 5 (ProOOL) [homepage on the Internet]. 2009 [updated 2012 Mar 21; cited 2020 Mar 10]. Available from: http://www.proqol.org.

6. Stamm, BH. The concise ProQOL manual. In The concise ProQOL mannual [homepage on the Internet]. 2nd ed. [updated 2010 Nov; cited 2020 Mar 10]. Available from: https://proqol.org/uploads/ProQOLManual.pdf

7. Khamisa N, Peltzer K, llic D, Oldenburg B. Work related stress, burnout, job satisfaction and general health of nurses: A follow-up study. Int J Nurs Pract. 2016;22(6):538-545. https://doi.org/10.1111/ijn.12455

8. Maslach C, Jackson SE. The measurement of experienced burnout. J Organ Behav. 1981;2(2):99-113. https://doi.org/10.1002/job.4030020205

9. Berger J, Polivka B, Smoot EA, Owens H. Compassion fatigue in pediatric nurses J Pediatr Nurs. 2015;30(6):e11-e17. https://doi.org/10.1016/j.pedn.2015.02.005

10. Hegney DG, Craigie M, Hemsworth D, et al. Compassion satisfaction, compassion fatigue, anxiety, depression and stress in registered nurses in Australia: Study 1 results. J Nurs Manag. 2014;22(4):506-518. https://doi.org/10.1111/jonm.12160

11. Hunsaker S, Chen HC, Maughan D, Heaston S. Factors that influence the development of compassion fatigue, burnout, and compassion satisfaction in emergency department nurses. J Nurs Scholarsh. 2015;47(2):186-194. https:// doi.org/10.1111/jnu.12122

12. Potter $P$, Deshields $T$, Divanbeigi J, et al. Compassion fatigue and burnout: Prevalence among oncology nurses. Clin J Oncol Nurs. 2010;14(5). https://doi. org/10.1188/10.CJON.E56-E62

13. Adeyemo SO, Omoaregba JO, Aroyewun BA, et al. Experiences of violence, compassion fatigue and compassion satisfaction on the professional quality of life of mental health professionals at a tertiary psychiatric facility in Nigeria. Open Sci J Clin Med. 2015;2(3):69-73.
14. Foster C. Investigating professional quality of life in nursing staff working in Adolescent Psychiatric Intensive Care Units (PICUs). J Ment Heal Training, Educ Pract. 2019;14(1):59-71. https://doi.org/10.1108/JMHTEP-04-2018-0023

15. Mashego TAB, Nesengani DS, Ntuli T, Wyatt G. Burnout, compassion fatigue and compassion satisfaction among nurses in the context of maternal and perinatal deaths. J Psychol Africa. 2016;26(5):469-472. https://doi.org/10.1080/ perinatal deaths. J Psycho Af

16. Mason HD, Nel JA. Compassion fatigue, burnout and compassion satisfaction: Prevalence among nursing students. J Psychol Africa. 2012;22(3):451-455. https://doi.org/10.1080/14330237.2012.10820554

17. Mathias CT, Wentzel DL. Descriptive study of burnout, compassion fatigue and compassion satisfaction in undergraduate nursing students at a tertiary education institution in KwaZulu-Natal. Curationis. 2017;40(1):1-6. https://doi.org/10.4102/ curationis.v40i1.1784

18. Wentzel DL, Brysiewicz P. A survey of compassion satisfaction, burnout and compassion fatigue in nurses practicing in three oncology departments in Durban, South Africa. Int J Africa Nurs Sci. 2018;8(December 2017):82-86. https://doi. org/10.1016/j.jjans.2018.03.004

19. Măirean C. Emotion regulation strategies, secondary traumatic stress, and compassion satisfaction in healthcare providers. J Psychol Interdiscip Appl. 2016;150(8):961-975. https://doi.org/10.1080/00223980.2016.1225659

20. Lauvrud C, Nonstad K, Palmstierna T. Occurrence of post traumatic stress symptoms and their relationship to professional quality of life (ProQoL) in nursing staff at a forensic psychiatric security unit: A cross-sectional study. Health Qual Life Outcomes. 2009;7:1-6. https://doi.org/10.1186/1477-7525-7-31

21. Mangoulia P, Koukia E, Alevizopoulos G, Fildissis G, Katostaras T. Prevalence of secondary traumatic stress among psychiatric nurses in Greece. Arch Psychiatr Nurs. 2015;29(5):333-338. https://doi.org/10.1016/j.apnu.2015.06.001

22. García-Izquierdo M, Ríos-Rísquez MI. The relationship between psychosocial job stress and burnout in emergency departments: An exploratory study. Nurs Outlook. 2012;60(5):322-329. https://doi.org/10.1016/j.outlook.2012.02.002

23. Adams RE, Boscarino JA, Figley CR. Compassion fatigue and psychological distress among social workers: A validation study. Am J Orthopsychiatry. 2006; 76(1):103-108. https://doi.org/10.1037/0002-9432.76.1.103

24. Duarte J. Professional quality of life in nurses: Contribution for the validation of the Portuguese version of the Professional Quality of Life Scale-5 (ProQOL-5). Análise Psicológica. 2017;35(4):529-542. https://doi.org/10.14417/ap.1260

25. Simpson B, Chipps J. Mental health legislation: Does it protect the rights of people with mental health problems? Soc Work. 2012;48(1):47-57. https://doi org/10.15270/48-1-104

26. Sacco TL, Ciurzynski SM, Harvey ME, Ingersoll GL. Compassion satisfaction and compassion fatigue among critical care nurses. Crit Care Nurse. 2015;35(4):32-43. https://doi.org/10.4037/ccn2015392

27. The South African Nursing Council South Africa. Information regarding the phasing out of legacy qualifications; And implementation of nursing qualifications aligned to the higher education qualifications sub-framework (HEQSF) [home page on the Internet]. [updated $2016 \mathrm{Dec} 20$; cited $2020 \mathrm{Apr} 09$ ]. Available from: https://www. sanc.co.za/archive/archive2016/newsc1607.htm

28. SANC. Circular 7. Phasing out of Legacy Qualifications and implementation of Nursing Qualifications aligned to the Higher Education Qualifications Sub-Framework (HEQSF). 2017;1978(50).

29. Rothmann S, Van der Colff JJ, Rothmann JC. Occupational stress of nurses in South Africa. Curationis. 2006;29(2):22-33. https://doi.org/10.4102/curationis. v29i2.1069

30. Franken $\mathrm{H}$, Parker J, Allen R, Wicomb RA. A profile of adult acute admissions to Lentegeur Psychiatric Hospital, South Africa. S Afr J Psychiatr. 2019;25:1-7. https://doi.org/10.4102/sajpsychiatry.v25i0.1244

31. Beck CT. Secondary traumatic stress in nurses: A systematic review. Arch Psychiatr Nurs. 2011;25(1):1-10. https://doi.org/10.1016/j.apnu.2010.05.005

32. Choi SH, Lee H. Workplace violence against nurses in Korea and its impact on professional quality of life and turnover intention. J Nurs Manag. 2017;25(7):508-518. https://doi.org/10.1111/jonm.12488

33. Dominguez-Gomez E, Rutledge DN. Prevalence of secondary traumatic stress among emergency nurses. J Emerg Nurs. 2009. https://doi.org/10.1016/j. jen.2008.05.003

34. Iyamuremye JD, Brysiewicz P. The development of a model for dealing with secondary traumatic stress in mental health workers in Rwanda. Heal SA Gesondheid. 2015;20(1):59-65. https://doi.org/10.1016/j.hsag.2015.02.006

35. Beth M, Makic F. Taking care of the caregiver: Compassion satisfaction and compassion fatigue. J Peri Anesthesia Nurs. 2015:30(6):546-547. https://doi. org/10.1016/j.jopan.2015.09.006

36. Chipps J, Ramlall S. Research in lower middle income countries-Recommendations for a national mental health research agenda in South Africa. Afr J Psychiatry. 2012;15(6):432-435. https://doi.org/10.4314/ajpsy.v15i6.55

37. Kim K, Han Y, Kwak Y, Kim J. Professional quality of life and clinical competencies among Korean nurses. Asian Nurs Res (Korean Soc Nurs Sci). 2015;9(3):200-206. https://doi.org/10.1016/j.anr.2015.03.002

38. Galiana L, Arena F, Oliver A, Sansó N, Benito E. Compassion satisfaction, compassion fatigue, and burnout in Spain and Brazil: ProQOL Validation and crosscultural diagnosis. J Pain Symptom Manage. 2017;53(3):598-604. https://doi.org/ 10.1016/j.jpainsymman.2016.09.014 